\title{
State of Health Monitoring in Machining: Extended Kalman Filter for Tool Wear Assessment in Turning of IN718 Hard-to-Machine Alloy
}

\author{
Farbod Akhavan Niaki ${ }^{1 *}$, Martin Michel $^{2}$ and Laine Mears ${ }^{3}$ \\ ${ }^{1}$ Clemson University International Center for Automotive Research, Greenville, S.C., U.S.A \\ ${ }^{2}$ Umwelt Campus Birkenfeld, Birkenfeld, Germany \\ ${ }^{3}$ Clemson University International Center for Automotive Research, Greenville, S.C., U.S.A \\ fakhava@clemson.edu,s12a29@umwelt-campus.de,mears@clemson.edu
}

\begin{abstract}
An Extended Kalman Filter (EKF) is employed in this work for tracking tool flank wear area in wetturning of Inconel 718 (IN718) nickel-based alloy in variable feed condition. The tool wear area evolution is modeled with a $3^{\text {rd }}$ order polynomial empirical function and an analytical solution for discrete state space system is derived. The state uncertainty was found to decrease up to a critical range of 200-250 $\mu \mathrm{m}$ of average flank wear length, and then increase abruptly with an increase in tool wear. Therefore, the tool wear uncertainty was modeled with failure probability density, i.e. the bathtub function, while a constant uncertainty was considered for the measurement signal (spindle power). To demonstrate the significance of using this method, the root mean square error (RMSE) and the mean absolute error (MAE) were calculated and compared with deterministic method in estimation of the tool wear area. It was shown that the proposed estimation based on stochastic filter EKF increased the accuracy of estimation by maximum of $60 \%$. Results for estimation of the rate of tool wear area indicate additional possible effects or transitions of effects at higher wear conditions.
\end{abstract}

Keywords: Extended Kalman Filter, Nickel-based alloy, Estimation, Uncertainty

\section{Introduction}

Monitoring the cutting tool performance during machining operations is a critical factor since the quality of the end-product and productivity rate are highly dependent on the functional state of the tool. A worn-out tool can deteriorate surface quality and causes high tensile residual stress which increases the possibility of micro-crack nucleation and early failure. Nickel-based alloys are a class of hard-to-machine materials that exhibit a combination of maintaining high strength at elevated

\footnotetext{
* Corresponding Author
} 
temperature and corrosion/creep resistance. These features make them a suitable candidate for high temperature/load environments such as the power generation industry. Nevertheless, the same features making them appealing candidates for such industries give them poor machinability features in terms of high tool wear rate and frequent tool change. As an example, in seven hours rough and fine cutting of a Ni-based blade, more than 40 cutting tools are needed to complete the process [1]. Therefore, monitoring the state of the tool in machining Ni-based alloys is significantly important to increase the productivity rate and reduce the machining downtime.

One of the challenges in tool wear monitoring is the complexity of its dynamics, and quantifying the effect of various variables such as tool coating, tool geometry, material structure, lubrication, tool run-out and initial residual stresses. Since controlling all these parameters is impossible, they act as the sources of uncertainties in machining. Therefore a stochastic-based method is required for analyzing the state of the tool. One of the early works in this field is the work of Schmitz et al. on stochastic estimation based on mesh grid method for identification of unknown parameters in 2-D Merchant model. The identified parameters were fed into the model for predicting cutting force and its uncertainty and experimentally validated on turning AISI-1045 steel [2]. In another effort by Karandikar et al., the authors used the mesh grid method and Markov Chain Monte Carlo (Metropolis algorithm) for Bayesian parameter inference on Taylor's tool life and extended Taylor's life in milling of AISI-4137 steel. They compared the results with deterministic approach (maximum likelihood estimation) and showed that by using the Bayesian method and combining the prior knowledge to the likelihood function, fewer experiments were required for parameter inference [3,4]. The performance of Bayesian inference and its superiority over deterministic approaches when limited information is available was also investigated on mechanistic tool wear model in milling Ren-108 Ni-based alloys [5]. Online stochastic estimation with the Kalman filter and the Particle Filter approach was also studied on the same material by Akhavan Niaki et al. They showed that by using the random sampling-based method such as the Particle Filter, a more informative prior can be selected for online parameter inference [6]. An alternative stochastic approach for tool wear studies is based on reliability and injury theory. Salonitis and Kolios investigated the applicability of using Monte Carlo simulation and first order reliability method for characterizing the probability of tool failure in different feed and cutting speed [7]. In an interesting work by Braglia and Catellano and Braglia et al., they derived the distribution of the tool life based on progressive behavior of the tool wear with diffusion theory and Fokker-Plank equation. They calculated the average and the uncertainty of progressive tool wear which were in agreement with experimental results $[8,9]$. While diffusion theory is successful in tracking progressive tool wear, it cannot be used for chipping or breakage detection; this will limit the applicability of their method.

The objective of this work is first to understand how uncertainties of the tool wear evolve over machining time and how to quantify these uncertainties. Second is to accurately estimate the progressive tool flank wear and mitigate the noise using a stochastic-based filter in turning of an IN718 Ni-based alloy. The organization of this work is as follows. The theoretical background of the stochastic-based estimation method used in this work will be discussed in section 2, followed by experimental setup in section 3. In section 4, uncertainty quantification in the state and the measurement models are addressed and results and conclusions are discussed in sections 5 and 6.

\section{Theoretical Background}

Assuming a Gaussian distribution for the states of a dynamic system, Rudolf Kálmán introduced a stochastic method of estimation as the Kalman filter [10]. Since then, the Kalman filter has been used extensively as an optimal control-observer for state estimation of linear and noisy dynamical systems $[6,11]$ For state estimation, the Kalman filter uses a closed-form discrete state space equation for linear systems and an approximation solution for nonlinear systems known as Extended Kalman filter 
(EKF). In the EKF, the nonlinear state or measurement model is linearized first and then the Kalman filter is applied for updating the mean and variance of the states. Depending on the system's degree of nonlinearity, the EKF might not be accurate. In this case, deterministic sampling methods such as Unscented Kalman Filters (UKF) [12] or random sampling methods such as Particle Filters (PF) [13] are proposed.

Assuming a discrete nonlinear state and measurement functions as $f$ and $g$, the state space representation of the system can be written as Equations 1 and 2; where $k$ is the time step, $x$ is the state's vector, $w$ is the state noise, $v$ is the measurement noise and $y$ is a measurement. In the context of machining, $x$ will be the tool wear; $y$ will be the sensor measurement.

$$
\begin{gathered}
x_{k}=f\left(x_{k-1}\right)+w_{k-1} \\
y_{k}=g\left(x_{k}\right)+v_{k}
\end{gathered}
$$

The states and measurements models $f$ and $g$ can be approximated by the $1^{\text {st }}$ order Taylor's expansion into Equations 3 and 4, where $\hat{x}$ is a posterior estimate of the state $x$, and $J$ and $G$ are Jacobians of functions $f$ and $g$ with respect to state $x$. These Jacobians are shown in Equations 5.

$$
\begin{gathered}
x_{k} \approx f\left(\hat{x}_{k-1}\right)+\left.J\right|_{\hat{x}_{k-1}}\left(x_{k-1}-\hat{x}_{k-1}\right)+w_{k-1} \\
y_{k} \approx g\left(\hat{x}_{k}\right)+\left.G\right|_{\hat{x}_{k}}\left(x_{k}-\hat{x}_{k}\right)+v_{k} \\
\left.J_{i j}\right|_{\hat{x}_{k-1}}=\frac{\partial f_{i}}{\partial x_{j}}\left(\hat{x}_{k-1}\right),\left.G_{i j}\right|_{\hat{x}_{k}}=\frac{\partial g_{i}}{\partial x_{j}}\left(\hat{x}_{k}\right)
\end{gathered}
$$

Using the linear Equations 3 and 4, Kalman filter can be applied as described by time update (Equations 6-7) and measurement update (Equations 8-10).

Time Update:

$$
\begin{gathered}
\hat{x}_{k}^{-}=f\left(\hat{x}_{k-1}\right) \\
P_{k}^{-}=\left.\left.J\right|_{\hat{x}_{k-1}} P_{k-1} J^{T}\right|_{\hat{x}_{k-1}}+Q_{k-1}
\end{gathered}
$$

Measurement Update:

$$
\begin{gathered}
K_{k}=\left.P_{k}^{-} G^{T}\right|_{\hat{x}^{-}}\left(\left.\left.G\right|_{\hat{x}^{-}} P_{k}^{-} G^{T}\right|_{\hat{x}^{-}}+R_{k}\right)^{-1} \\
\hat{x}_{k}=\hat{x}_{k}^{-}+K_{k}\left(y_{k}-g\left(\hat{x}_{k}^{-}\right)\right) \\
P_{k}=\left(I-\left.K_{k} G^{T}\right|_{\hat{x}^{-}}\right) P_{k}^{-}
\end{gathered}
$$

\section{Experimental Setup}

The work was conducted on annealed IN718 Ni-based alloys which contain $53.8 \% \mathrm{Ni}, 18.44 \% \mathrm{Cr}$, $17.33 \% \mathrm{Fe}, 5.31 \% \mathrm{Nb}+\mathrm{Ta}, 0.97 \% \mathrm{Ti}, 0.58 \mathrm{Al}$ and less than $0.1 \%$ of other elements, with $94 \mathrm{HRB}$ hardness. The OKUMA CNC lathe was used for turning $50 \mathrm{~mm}$ of an IN718 bar in a wet cutting condition with 6\% concentration. The insert used was Sandvik CNGG 120404 SGF PVD coated insert with $0.4 \mathrm{~mm}$ tool nose radius shown in Figure 1. To investigate the effect of variable cutting conditions on the process, feed was changed between $0.05 \mathrm{~mm} / \mathrm{rev}$ to $0.15 \mathrm{~mm} / \mathrm{rev}$ while cutting speed 
and depth of cut were kept constant at $80 \mathrm{~m} / \mathrm{min}$ and $0.1 \mathrm{~mm}$ as recommended by Sandvik. A Design of Experiment (DoE) table used in this work is shown in Table 1. Four replications of feeds $0.05,0.1$ and $0.15 \mathrm{~mm} / \mathrm{rev}$ were used for model development and uncertainty quantification while other feeds were used for validation purposes.
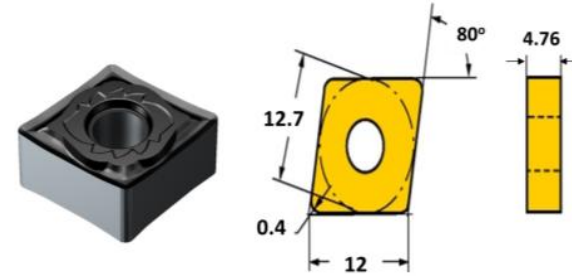

Figure 1: Sandvik CNGG 120404 SGF insert
Table 1: Design of Experiment table for turning IN718

\begin{tabular}{cccc}
\hline $\begin{array}{c}\text { Cutting Speed } \\
(\mathrm{m} / \mathrm{min})\end{array}$ & $\begin{array}{c}\text { Feed } \\
(\mathrm{mm} / \mathrm{rev})\end{array}$ & $\begin{array}{c}\text { Depth of } \\
\text { cut }(\mathrm{mm})\end{array}$ & Replications \\
\hline 80 & 0.050 & 0.1 & 4 \\
80 & 0.100 & 0.1 & 4 \\
80 & 0.150 & 0.1 & 4 \\
80 & 0.063 & 0.1 & 1 \\
80 & 0.088 & 0.1 & 1 \\
80 & 0.113 & 0.1 & 1 \\
80 & 0.138 & 0.1 & 1 \\
\hline
\end{tabular}

As shown by Rubenstein in his work, cutting force (and consequently cutting power) is dependent on the area of the wear on the flank face [14]; therefore the focus of this study is on tracking evolution of this parameter. Each replication starts with a sharp insert and the area of wear land on the flank side (shown in Figure 2) was measured after each experiment (cutting 50mm length). It was decided to continue the operation beyond the $300 \mu \mathrm{m}$ tool wear width suggested by ISO-8688 to investigate the effect of excessive tool wear on surface quality, dimensional integrity and residual stress of the workpiece. Spindle power consumption was collected using an Hall effect sensor with $100 \mathrm{~Hz}$ sampling rate with NI-cRIO9103. Then the mean value of the signal between $85 \%-95 \%$ of the cutting length was calculated as measurement signal $(y)$. The results of the tool wear area and spindle power measurement for all the replications of the feeds $0.05,0.1$ and $0.15 \mathrm{~mm} / \mathrm{rev}$ is shown in Figure 3 to Figure 5. The lowest variation in the tool flank wear area belongs to the lowest feed $(0.05 \mathrm{~mm} / \mathrm{rev})$ and the largest variation belongs to the mid-feed $(0.1 \mathrm{~mm} / \mathrm{rev})$ where significant departure observed after the reaching the wear area of $0.1 \mathrm{~mm}^{2}$ (approximately $200 \mu \mathrm{m}$ of average tool wear length).
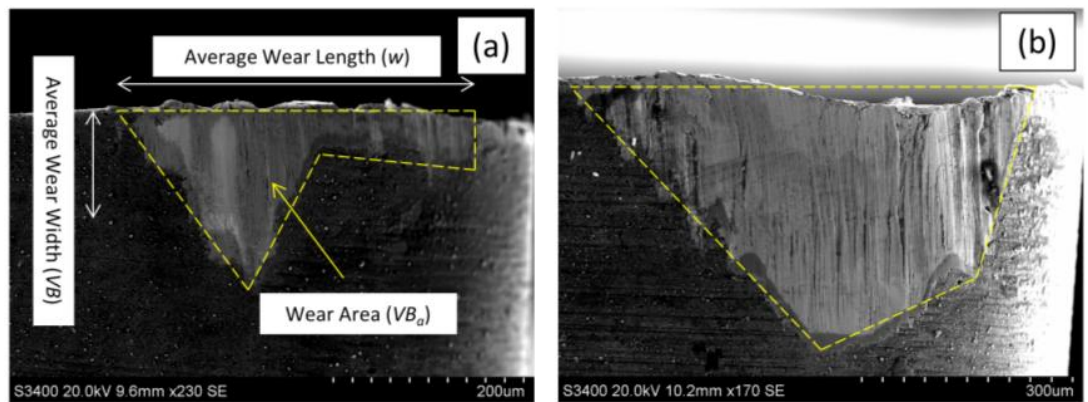

Figure 2: Scanning Electron Microscopy (SEM) image of flank wear land with feed $0.1 \mathrm{~mm} / \mathrm{rev}$, cutting speed $80 \mathrm{~m} / \mathrm{min}$ and depth of cut $0.1 \mathrm{~mm}$ (a) initial wear and (b) dull tool 

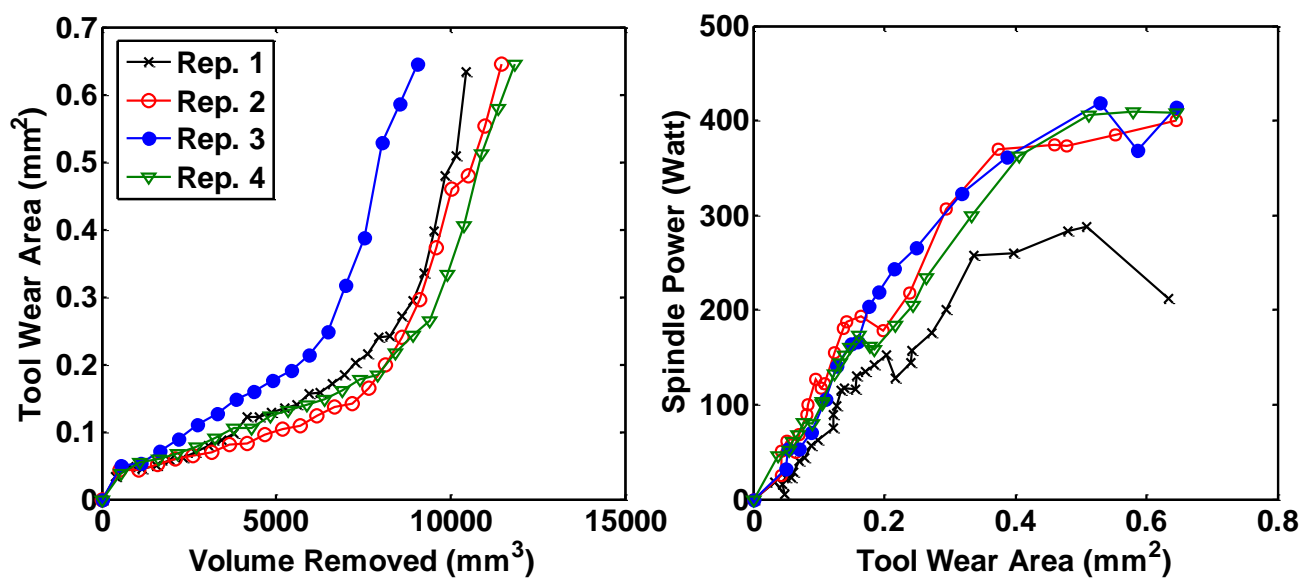

Figure 3: Tool wear area (left) and Spindle power (right) for feed of $0.05 \mathrm{~mm} / \mathrm{rev}$
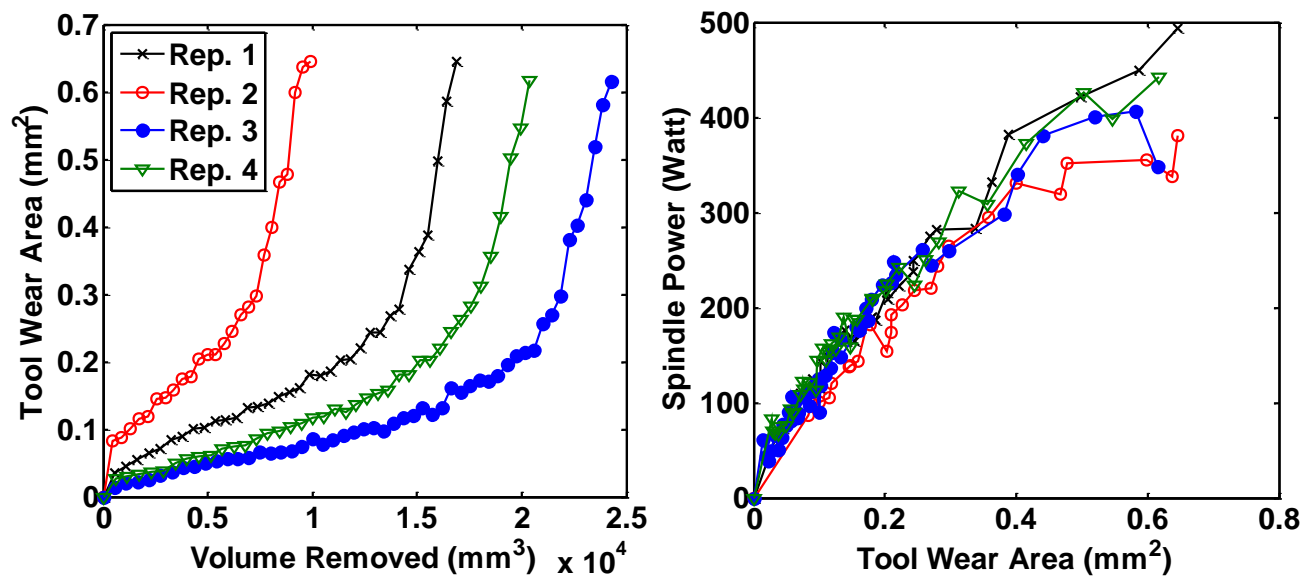

Figure 4: Tool wear area (left) and Spindle power (right) for feed of $0.10 \mathrm{~mm} / \mathrm{rev}$
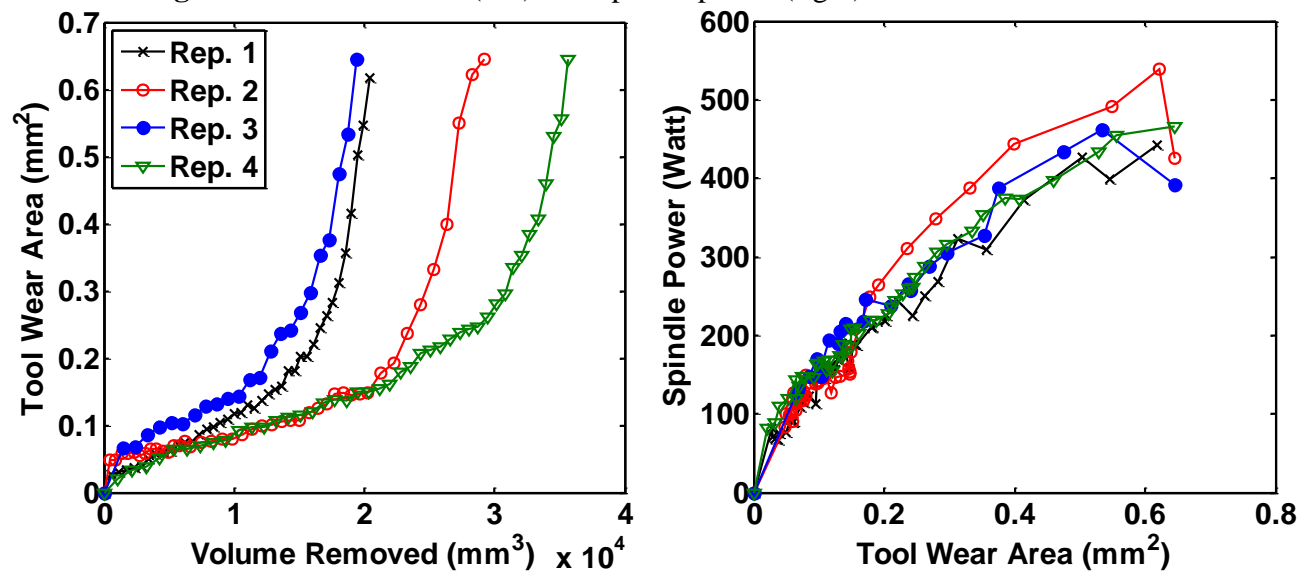

Figure 5: Tool wear area (left) and Spindle power (right) for feed of $0.15 \mathrm{~mm} / \mathrm{rev}$ 


\section{Stochastic State and Measurement Models}

The tool wear area in the Figure 3 to Figure 5 can be represented by an empirical $3^{\text {rd }}$ order polynomial function with more than $95 \% R_{a d j}^{2}$ as goodness of fit shown in Equation 11, where $M R$ denotes material removed, $a, b$ and $c$ are the feed-dependent polynomial coefficients and $V B_{a}$ is the flank wear area. By taking derivate of this function the $V B_{a}$ rate can be found as $2^{\text {nd }}$ order polynomial shown in Equation 13. However, to write the state space model, the trajectory of the state $V B_{a}$ is required, i.e. the parameter $M R$ should be eliminated from the Equation 11 and 12 and the $V B_{a}$ rate should be written as function of $V B_{a}$.

$$
\begin{gathered}
V B_{a}=a M R^{3}+b M R^{2}+c M R \\
V B_{a}^{\prime}=3 a M R^{2}+2 b M R+c
\end{gathered}
$$

In the $16^{\text {th }}$ century, Gerolamo Cardano found a solution for solving cubic functions. Using the Cardano's formula, a closed-form solution for Equation 11 was found and substituted into the Equation 12. After some simplifications Equations 13 to 15 can be derived representing continuous function of $V B_{a}$ rate and $V B_{a}$. In these equations, $w$ represents the added normally distributed noise.

$$
\begin{gathered}
V B_{a}^{\prime}=3 a\left(\alpha^{\frac{2}{3}}+\beta^{\frac{2}{3}}\right)-2 a A+c-\frac{b^{2}}{3 a}+w \\
\alpha=-\frac{B}{2}+\sqrt{\frac{B^{2}}{4}+\frac{A^{3}}{27}}, \beta=\frac{B}{2}+\sqrt{\frac{B^{2}}{4}+\frac{A^{3}}{27}} \\
A=\frac{c}{a}-\frac{b^{3}}{3 a^{2}}, B=\frac{-V B_{a}}{a}+\frac{2 b^{3}}{27 a^{3}}-\frac{b c}{3 a^{2}}
\end{gathered}
$$

Writing the $V B_{a}$ rate in Equation 13 as $\frac{V B_{a, k}-V B_{a, k-1}}{\Delta M R}$, the discretized nonlinear state function can be written as Equations 16. The last step is linearizing this equation by taking the Jacobian of the nonlinear function $f$. This is shown in Equation 17 and 18.

$$
\begin{aligned}
& V B_{a, k}=\Delta M R_{k}\left(3 a\left(\alpha_{k-1}^{\frac{2}{3}}+\beta_{k-1}^{\frac{2}{3}}\right)-2 a A+c-\frac{b^{2}}{3 a}\right)+V B_{a, k-1}+\Delta M R_{k} w_{k} \\
& =f\left(V B_{a, k-1}\right)+\Delta M R_{k} w_{k} \\
& J_{k}=\frac{\partial f}{\partial V B_{a, k-1}}=2 a \cdot \Delta M R_{k-1}\left(\alpha_{k-1}^{-\frac{1}{3}} \alpha_{k-1}^{\prime}+\beta_{k-1}^{-\frac{1}{3}} \beta_{k-1}^{\prime}\right)+1 \\
& \alpha_{k}^{\prime}=\frac{1}{2 a}\left(1-\frac{B_{k}}{2}\left(\frac{B_{k}^{2}}{4}+\frac{A^{3}}{27}\right)^{-\frac{1}{2}}\right), \beta_{k}^{\prime}=\frac{1}{2 a}\left(-1-\frac{B_{k}}{2}\left(\frac{B_{k}^{2}}{4}+\frac{A^{3}}{27}\right)^{-\frac{1}{2}}\right)
\end{aligned}
$$

The next step is developing the relationship of the tool wear area and the spindle power. An analytical solution suggested by Rubenstein based on shape and properties of adhered asperities on the flank face of the tool is used in this work [14]. As discussed in Rubenstein's work, the cutting force can be written as Equation (19), where $F_{c^{\prime}}$ is a constant for a given tool, geometry and cutting parameters, $\mu$ is friction coefficient assumed equal to 0.1 in wet cutting, $H_{m}$ is the hardness of the tool, 
$w$ is wear length, $V B$ is wear width and $c_{l}$ is a constant. The product of wear length and width produces the wear land area $\left(V B_{a}\right)$. The cutting power is simply derived by the product of cutting force $\left(F_{c}\right)$ and cutting speed $\left(V_{c}\right)$ which is kept constant in all of the experiments of this work.

$$
F_{c}=F_{c^{\prime}}+c_{1} \mu H_{m} w V B \rightarrow P=F_{c^{\prime}} V_{c}+c_{1} V_{c} \mu H_{m} w \times V B \text { where }\left(w \times V B=V B_{a}\right)
$$

The product of parameters $c_{l}, V_{c}, \mu$ and $H_{m}$ are constants and can be found as the average slope of spindle power versus tool wear area curves. The parameter $F_{c^{\prime}}$ of Equation (19) was considered to be feed dependent. Therefore the product of this parameter to cutting speed represents the amount of power required to cut the material when using a sharp insert. To find the relationship, 4 replications of tests with sharp inserts in 5 different feeds were conducted and a linear model with $93 \% R^{2}$ was fitted to the data accordingly. The measured results are shown in Table 2 and Figure 6 and the measurement model is described in Equation 20 where $f_{e}$ is representing feed. Note that the cutting speed is constant $(=80 \mathrm{~m} / \mathrm{min})$ throughout all the experiments.

Table 2: Effect of different feeds on spindle power when sharp tool is used

\begin{tabular}{cccccc}
\hline $\begin{array}{c}\text { Power }(\text { Watt }) \\
=F_{c^{\prime}} \times V_{c}\end{array}$ & \multicolumn{5}{c}{ Feed $-\boldsymbol{f}_{\boldsymbol{e}}(\mathbf{m m} / \mathbf{r e v})$} \\
& $\mathbf{0 . 0 5}$ & $\mathbf{0 . 0 7 5}$ & $\mathbf{0 . 1}$ & $\mathbf{0 . 1 2 5}$ & $\mathbf{0 . 1 5}$ \\
$\mathrm{P}_{1}$ & 33.6 & 38.6 & 59.2 & 48.0 & 113.8 \\
$\mathrm{P}_{2}$ & 24.5 & 55.6 & 74.4 & 71.7 & 91.7 \\
$\mathrm{P}_{3}$ & 39.2 & 41.1 & 59.1 & 83.1 & 122.9 \\
$\mathrm{P}_{4}$ & 40.9 & 71.7 & 84.1 & 81.4 & 83.1 \\
Average & 34.56 & 51.73 & 69.22 & 71.07 & 102.87 \\
Standard Deviation & 7.39 & 15.29 & 12.26 & 16.16 & 18.59 \\
\hline
\end{tabular}

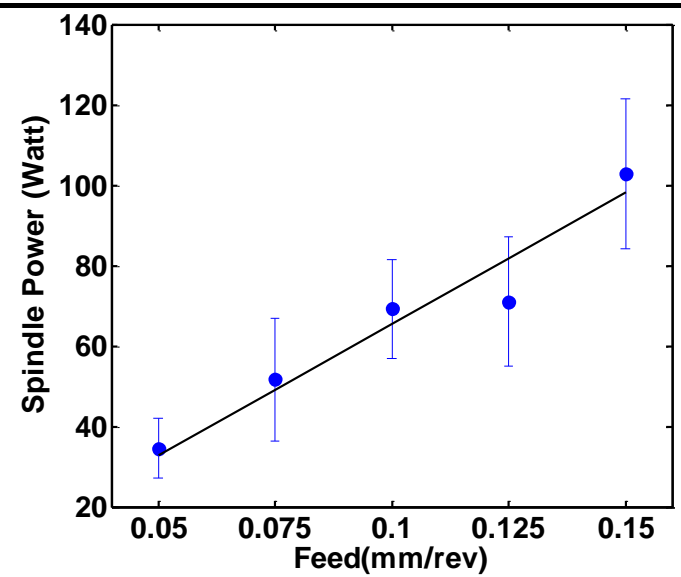

Figure 6: Spindle power change with feed when sharp insert is used

$$
P=644 V B_{a}+504 f_{e} V_{c} \stackrel{\text { discrete }}{\longrightarrow} P_{k}=644 V B_{a, k}+504 f_{e} V_{c}+v_{k}
$$

\subsection{Uncertainty Quantification for the State and Measurement Models}

Since the variation in the spindle power is relatively constant throughout the whole process (Figure 3 to Figure 5), the maximum standard deviation of the measured power of the 4 replications of each feed was calculated equal to 40 Watts. On the other hand, a different strategy should be taken to find the uncertainty for the state $V B_{a}$. Considering Figure 7(a), which shows the standard deviation in 
different feeds, an interesting fact emerged. The uncertainties in the tool wear area decrease at the beginning of the process and reach a relatively constant value around $V B_{a}=0.15 \mathrm{~mm}^{2}$ (equivalent to nearly $200 \mu \mathrm{m}$ of the average tool wear length). Then it begins to increase with an increase in the tool wear area, which consequently explains the large variation and departure. Uncertainty was calculated based on the bathtub curve model and is shown in Figure 7(b).

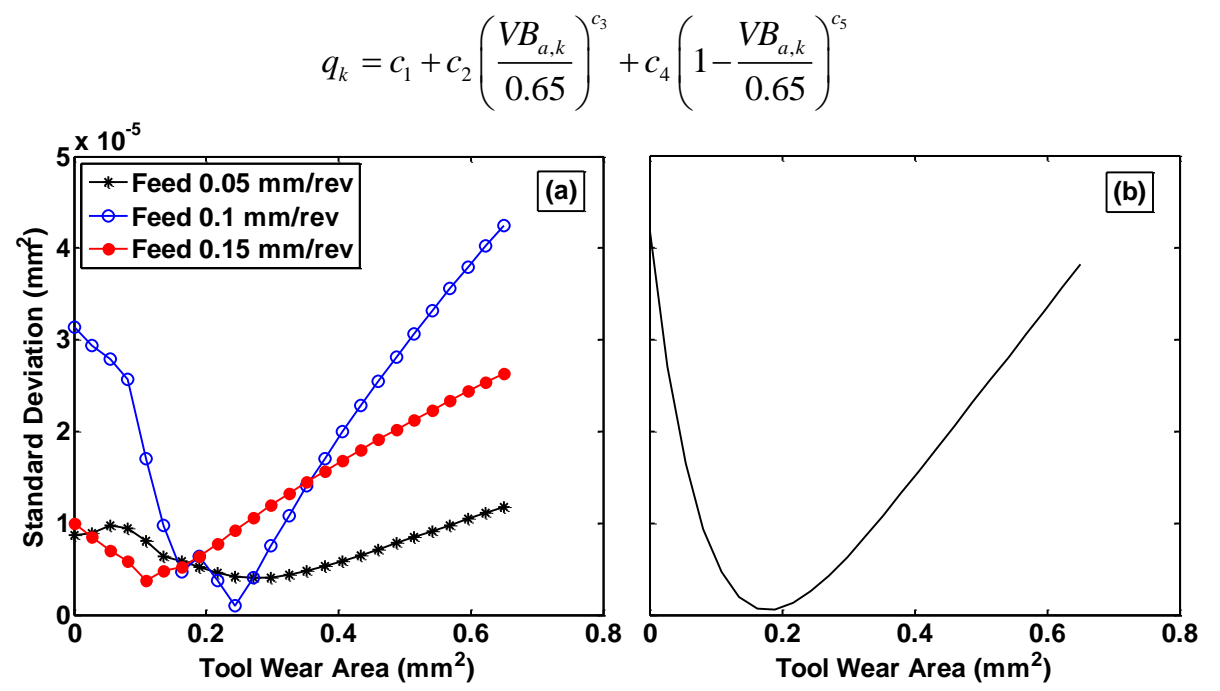

Figure 7: Uncertainties propagation (a) different feeds and (b) Modeled bathtub curve for state uncertainty function

Table 3: Identified coefficients of bathtub function based on simplex search algorithm

\begin{tabular}{cccccc}
\hline Model & \multicolumn{4}{c}{$q_{k}=c_{1}+c_{2}\left(\frac{V B_{a, k}}{0.65}\right)^{c_{3}}+c_{4}\left(1-\frac{V B_{a, k}}{0.65}\right)^{c_{5}}$} & \\
\hline $\boldsymbol{C}_{\boldsymbol{i}}$ & $C_{1}$ & $C_{2}$ & $C_{3}$ & $C_{4}$ & $C_{5}$ \\
Value & $-2.1 \mathrm{E}-05$ & $5.9 \mathrm{E}-05$ & 1.028 & $6.3 \mathrm{E}-05$ & 7.43 \\
\hline
\end{tabular}

The state space representation of the system is summarized as Equations 22 to 25 . Note that in Equation 22, $V B_{a, k-1}$ is embedded in $\alpha$ and $\beta$ parameters as well.

$$
\begin{gathered}
\text { State Model } \rightarrow V B_{a, k}=\Delta M R_{k}\left(3 a\left(\alpha_{k-1}^{\frac{2}{3}}+\beta_{k-1}^{\frac{2}{3}}\right)-2 a A+c-\frac{b^{2}}{3 a}\right)+V B_{a, k-1}+\Delta M R_{k} w_{k-1} \\
w_{k} \sim N\left(0, q_{k}^{2}\right) \text { where } q_{k}=\left(-2.1+5.9\left(\frac{V B_{a, k}}{0.65}\right)^{1.028}+6.3\left(1-\frac{V B_{a, k}}{0.65}\right)^{7.43}\right) \times 10^{-5} \\
\text { Measurment Model } \rightarrow P_{k}=644 V B_{a, k}+504 f_{e} V_{c}+v_{k} \\
v_{k} \sim N\left(0, r_{k}^{2}\right) \text { where } r_{k}=40
\end{gathered}
$$




\section{Results and Discussion}

To test the performance of the EKF in estimating the tool flank wear area, first its performance was tested on all the 4 replications for feeds $0.05,0.1$ and $0.15 \mathrm{~mm} / \mathrm{rev}$. The initial value of $\mathrm{VB}_{\mathrm{a} 0}$ is chosen as $0.04 \mathrm{~mm}^{2}$ with the initial variance of $0.007 \mathrm{~mm}^{4}$ for all the estimations. The resulting estimated mean (black curve) and uncertainty (red curve) for the tool flank are shown in Figure 8 to Figure 10.
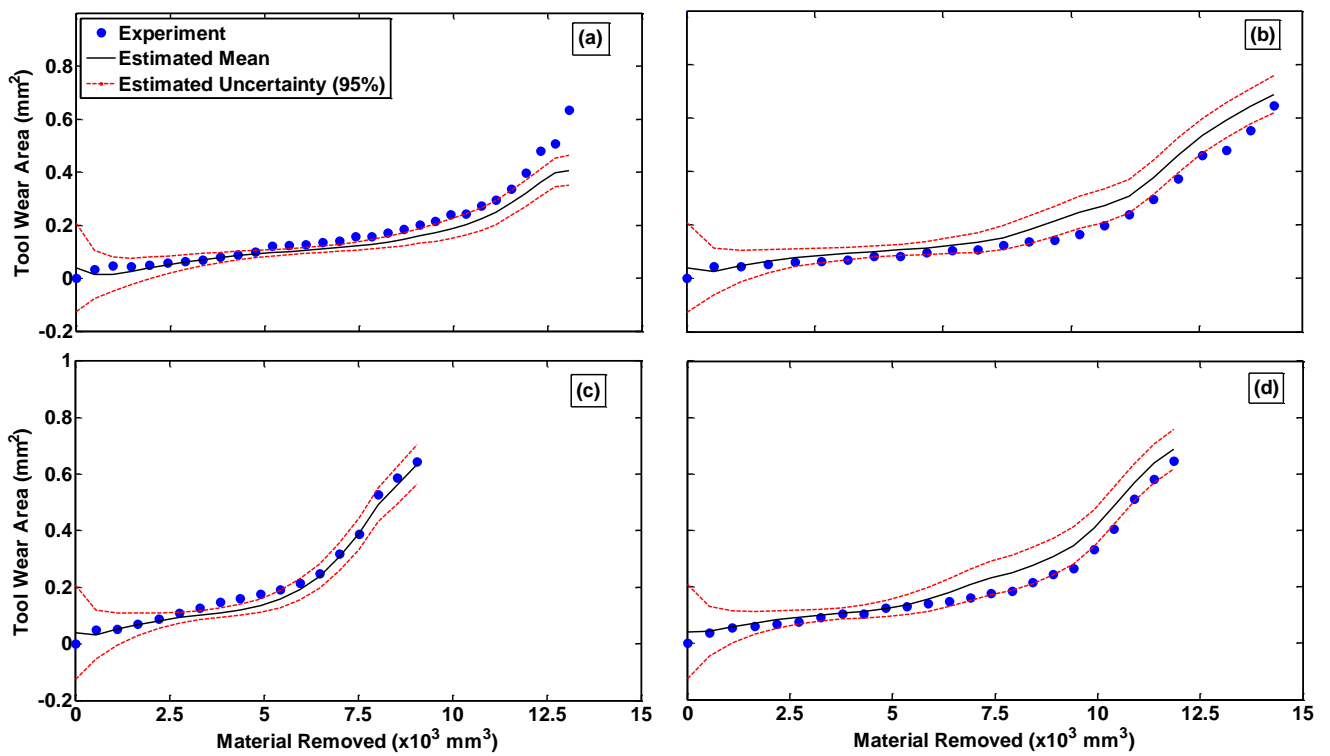

Figure 8: Estimated tool wear area for feed $0.05 \mathrm{~mm} / \mathrm{rev}$, (a) Replication 1, (b) Replication 2, (c) Replication
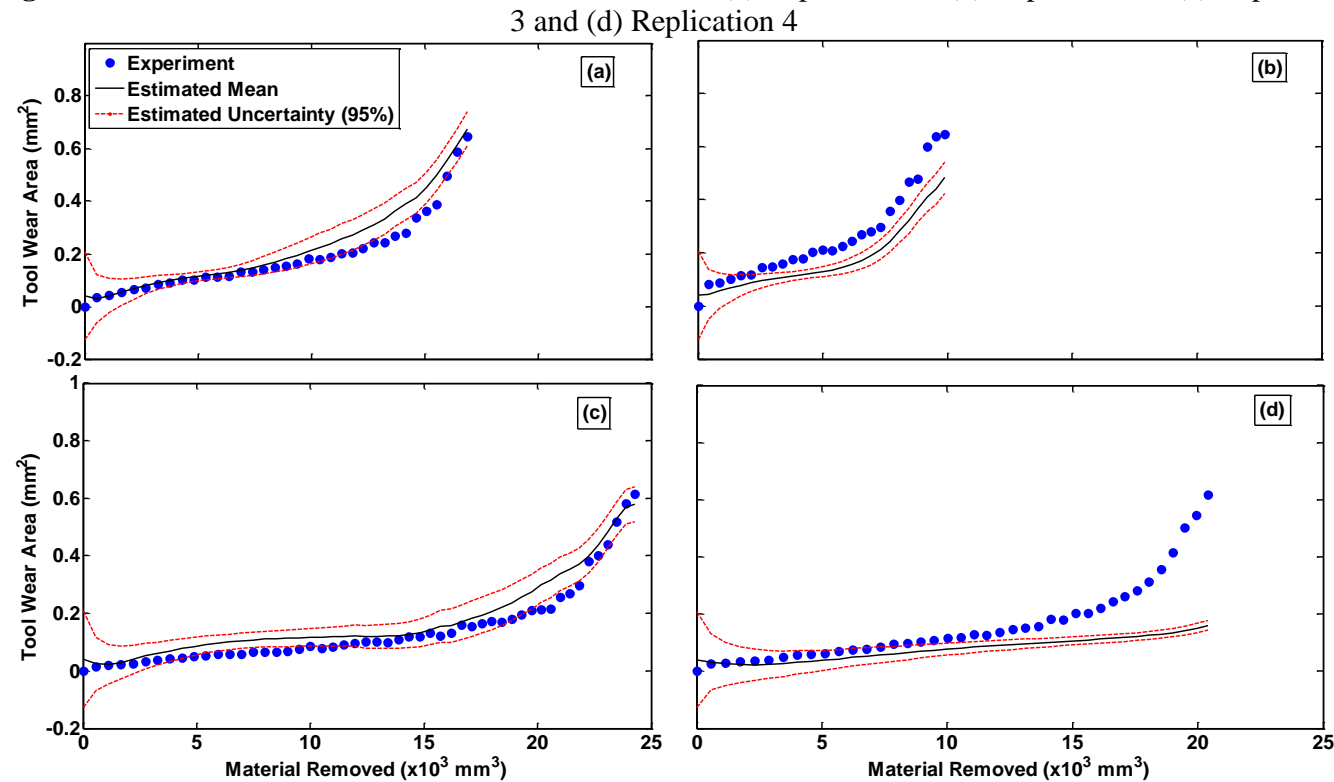

Figure 9: Estimated tool wear area for feed $0.10 \mathrm{~mm} / \mathrm{rev}$, (a) Replication 1, (b) Replication 2, (c) Replication 3 and (d) Replication 4 

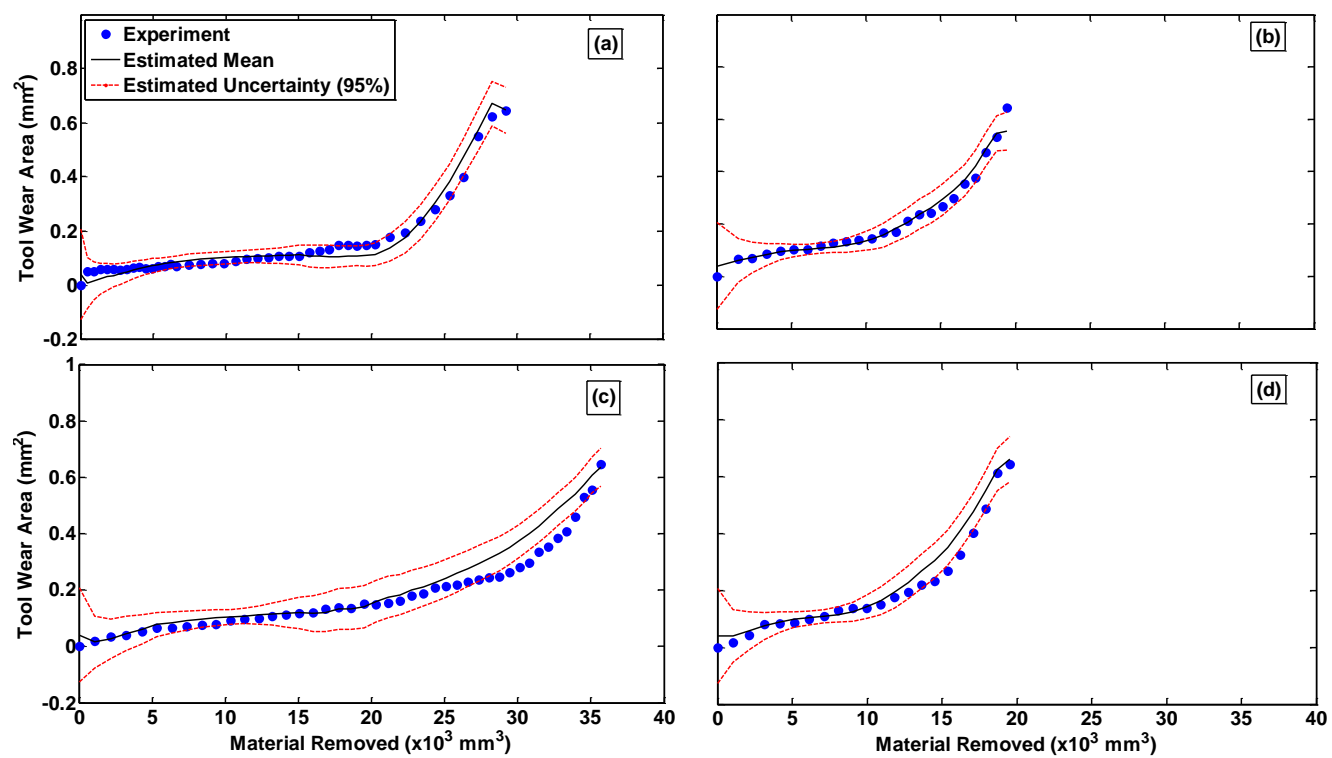

Figure 10: Estimated tool wear area for feed $0.15 \mathrm{~mm} / \mathrm{rev}$, (a) Replication 1, (b) Replication 2, (c)

Replication 3 and (d) Replication 4

According to the Figure 8 , in the $1^{\text {st }}$ replication, the EKF was able to estimate the tool wear area only up to $0.4 \mathrm{~mm}^{2}$ and was unable to predict accurately beyond that. This is due to the effect of measured power, which reduced abruptly as shown in Figure 3. The Root Mean Square Error (RMSE) of the $1^{\text {st }}$ replication was calculated as $0.06 \mathrm{~mm}^{2}$. The largest inaccuracy in estimation occurred in the feed $0.10 \mathrm{~mm} / \mathrm{rev}$ where in the $2^{\text {nd }}, 3^{\text {rd }}$ and $4^{\text {th }}$ replications the EKF failed to have an acceptable accuracy with maximum of $0.14 \mathrm{~mm}^{2}$ RMSE. This is due to the large variations that exist in experimental replications which make the state model less accurate. Finally, for feed $0.15 \mathrm{~mm} / \mathrm{rev}$, the EKF performed well except for the $3^{\text {rd }}$ replication with $0.05 \mathrm{~mm}^{2}$ RMSE. In all the other replications the experimental measurement fell in the $95 \%$ prediction interval of the filter.

To better assess the performance of the EKF, validation sets were used for estimating the progressive tool flank wear area. Figure 11 through Figure 14 show the results of the estimation. To have a closer look at the performance of the EKF in predicting tool wear and tool wear rate, the trajectory function (meaning tool wear area rate versus tool wear area) and the progressive tool wear function are shown side by side. As can be seen in these figures, the EKF is able to have an accurate estimation for the tool wear area with less than $0.04 \mathrm{~mm}^{2}$ RMSE, however there is still inaccuracy in estimating the tool wear area rate specifically after reaching $0.4 \mathrm{~mm}^{2}$. To demonstrate the significance of stochastic filter in improving estimation accuracy the RMSE and Mean Absolute Error (MAE) of the estimated tool wear area are compared in Figure 15 for all the 4 tests with a deterministic estimation which only uses the power-tool wear area relation shown in Equation 20 (without additive noise $v_{k}$ ). It can be seen from this figure that, the EKF estimation outperformed the deterministic method in 3 of the tests by $31 \%, 60 \%$ and $36 \%$ accuracy improvements which are significant in estimation results. 

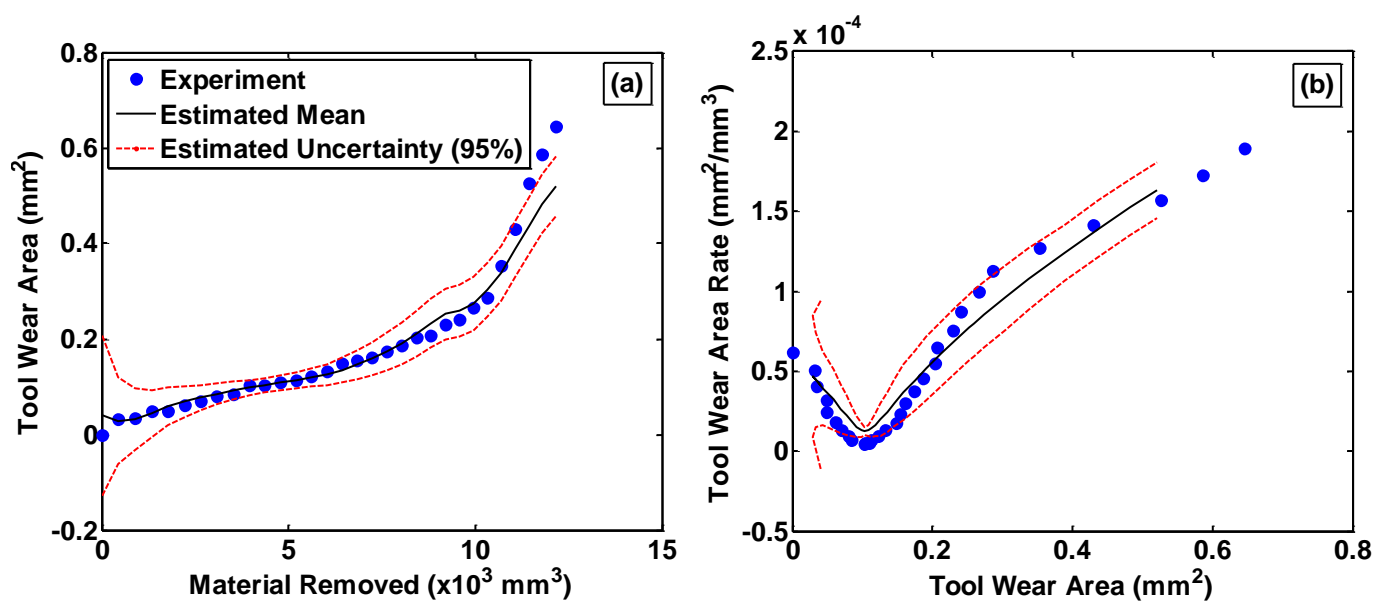

Figure 11: Estimated tool wear and tool wear rate for feed $0.0625 \mathrm{~mm} / \mathrm{rev}$, (a) Estimated tool wear area, (b) trajectory estimation
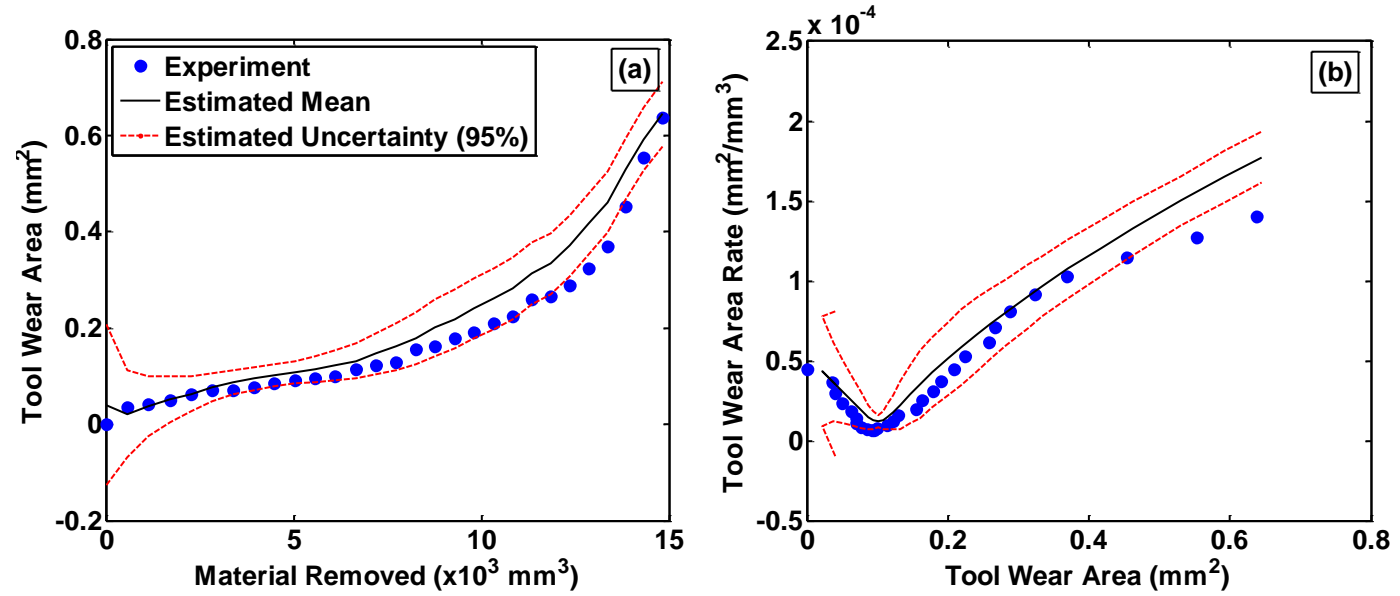

Figure 12: Estimated tool wear and tool wear rate for feed $0.0875 \mathrm{~mm} / \mathrm{rev}$, (a) Estimated tool wear area, (b) trajectory estimation
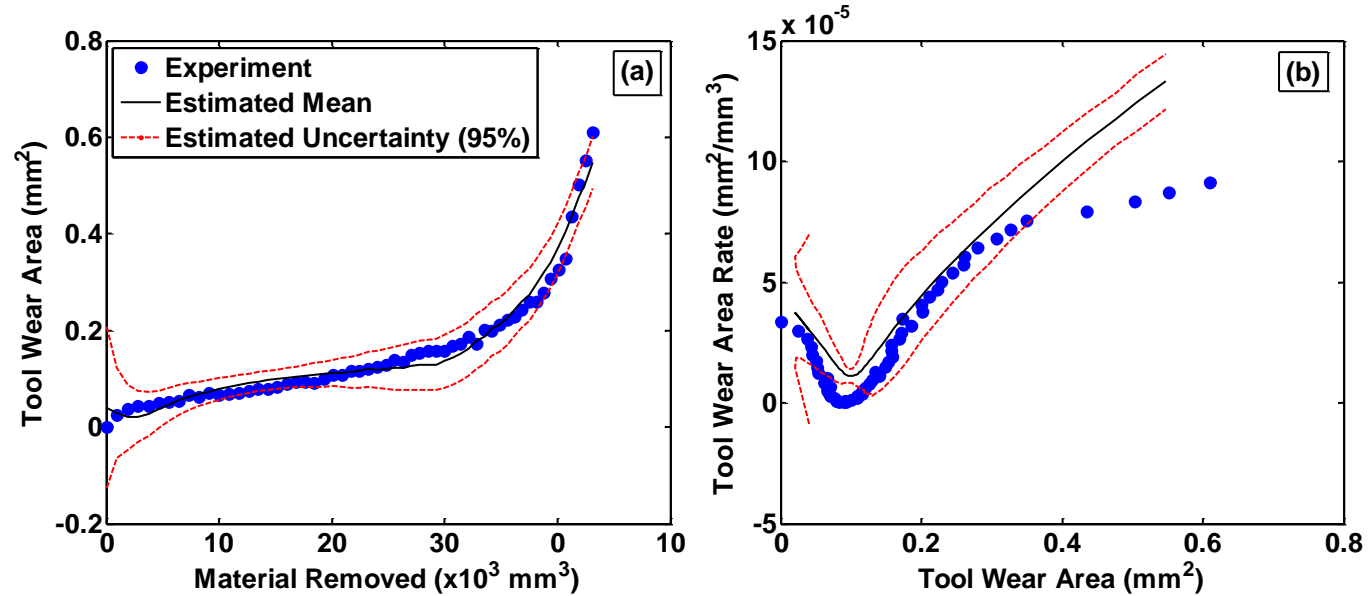

Figure 13: Estimated tool wear and tool wear rate for feed $0.1125 \mathrm{~mm} / \mathrm{rev}$, (a) Estimated tool wear area, (b) trajectory estimation 

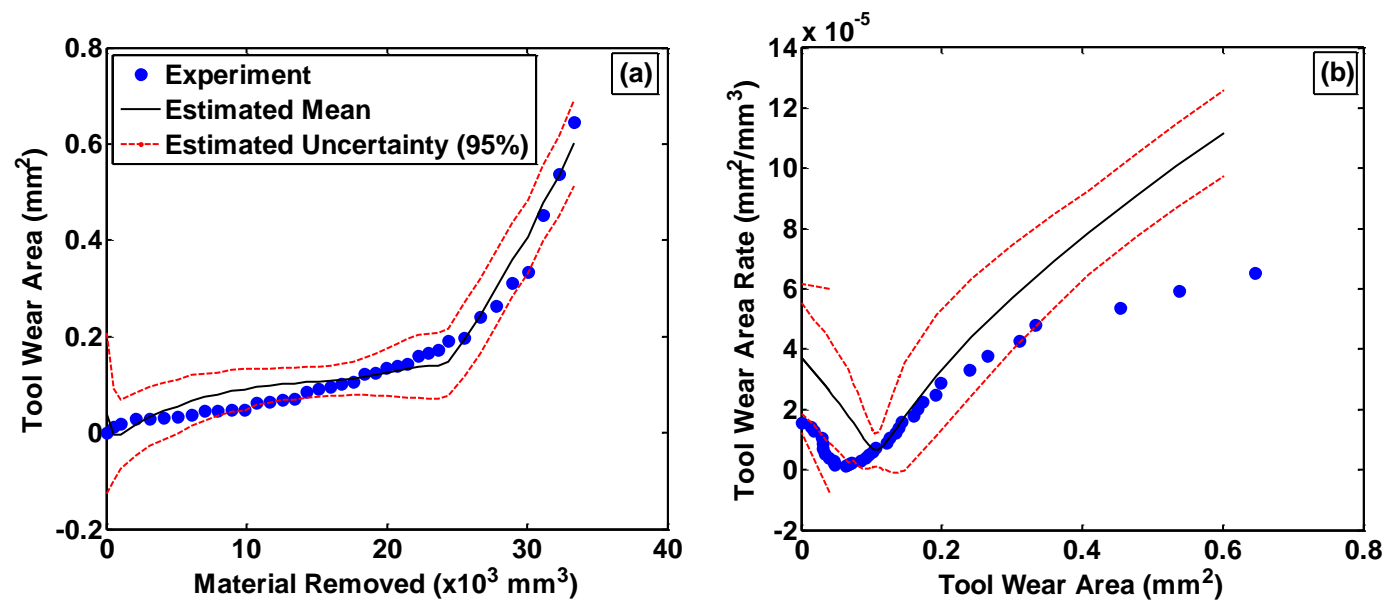

Figure 14: Estimated tool wear and tool wear rate for feed $0.1375 \mathrm{~mm} / \mathrm{rev}$, (a) Estimated tool wear area, (b) trajectory estimation
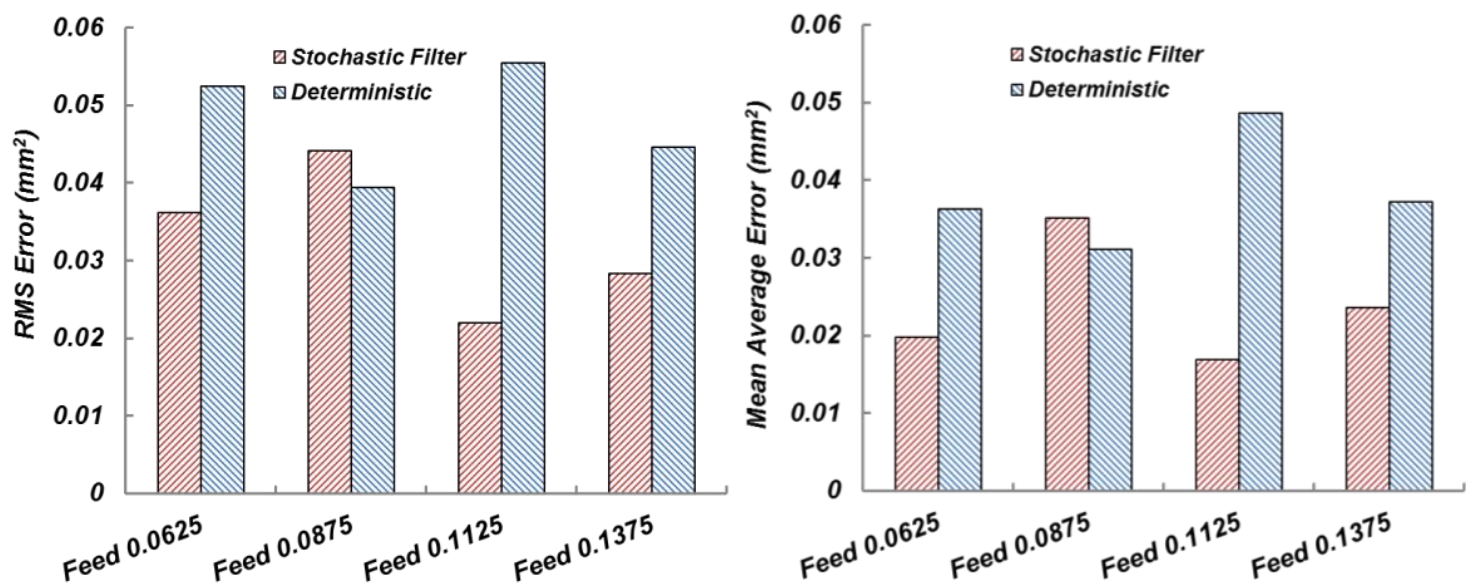

Figure 15: Error comparison in the estimated tool wear area for validation tests

\section{Conclusions}

Monitoring the performance of the tool in machining Ni-based alloys is a critical yet challenging task, since various sources of uncertainty exist in the operation. Therefore a stochastic vision of the estimated tool wear is required. The extended Kalman filter provides a robust framework for estimating states of the system in the presence of noise. This method was deployed in this study, and findings are summarized as following:

- A large number of experiments was conducted to quantify the uncertainty function in the tool wear. To increase the accuracy it was decided to measure the area of the flank wear instead of average flank wear length.

- The state and measurement models were found based on 4 replication sets of the feeds $0.05,0.1$ and $0.15 \mathrm{~mm} / \mathrm{rev}$. An analytical solution was derived for the nonlinear function of the state model in addition to a linear function for the measurement model. 
- It was observed that up to average tool wear length of $200-250 \mu \mathrm{m}$, the uncertainty decreases following by an increase beyond this value. The uncertainty in the state model was quantified with a failure probability function, modeled as a bathtub curve. Uncertainty in the measurement model was considered constant throughout all experiments.

- The EKF performance was tested and compared with deterministic method in 4 validation tests; less than $0.04 \mathrm{~mm}^{2}$ RMSE and maximum of $60 \%$ improvement in estimation accuracy were observed for the tool wear area estimation using EKF, and all the experimental results fell into the $95 \%$ prediction interval of the EKF. However, the EKF did not performed well in estimating the rate of tool wear area.

While monitoring the tool wear is critical in machining Ni-based alloys, its effect on the surface quality, surface residual stress or dimensional integrity of the workpiece has not been discussed in the literature. In other words, it is not clear how much damage certain amount of tool wear can cause to the dimensional integrity or surface roughness and how the uncertainties of the tool wear propagate into that. Therefore, a function representing the examination of all of these factors together can lead to a better decision making strategy, and provide a more informed solution on when to change the tool before damaging the quality of the end-product. This function will be introduced in future works.

\section{Acknowledgments}

The authors wish to thank the National Science Foundation for support of this work under Grant No. 0954318. Any opinions, findings, and conclusions or recommendations expressed in this material are those of the authors and do not necessarily reflect the views of the National Science Foundation.

\section{References}

[1] Zhu D, Zhang X, Ding H. Tool wear characteristics in machining of nickel-based superalloys. Int.J.Mach.Tools Manuf. 2013;64:60-77.

[2] Schmitz TL, Karandikar J, Kim NH, Abbas A. Uncertainty in machining: Workshop summary and contributions. Journal of Manufacturing Science and Engineering 2011;133:051009.

[3] Karandikar JM, Abbas AE, Schmitz TL. Tool life prediction using Bayesian updating. Part 1: Milling tool life model using a discrete grid method. Precis Eng 2014;38:9-17.

[4] Karandikar JM, Abbas AE, Schmitz TL. Tool life prediction using Bayesian updating. Part 2: Turning tool life using a Markov Chain Monte Carlo approach. Precis Eng 2014;38:18-27.

[5] Akhavan Niaki F, Ulutan D, Mears L. Parameter Inference under Uncertainty in End-Milling Gamma Prime Strengthened Difficult-to-Machine Alloy. Journal of Manufacturing Science and Engineering 2016.

[6] Akhavan Niaki F, Ulutan D, Mears L. Stochastic tool wear assessment in milling difficult to machine alloys. International Journal of Mechatronics and Manufacturing Systems 2015;8:134-159. 
[7] Salonitis K , Kolios A. Reliability assessment of cutting tools life based on advanced approximation methods. Procedia CIRP 2013;8:397-402.

[8] Braglia M , Castellano D. Diffusion theory applied to tool-life stochastic modeling under a progressive wear process. Journal of Manufacturing Science and Engineering 2014;136:031010.

[9] Braglia M, Castellano D, Frosolini M. Stochastic theory of tool life-Theoretical developments on the injury theory. International Journal of Mathematical Modelling and Numerical Optimisation 2014;5:265-279.

[10] Kalman RE. A new approach to linear filtering and prediction problems. Journal of Fluids Engineering 1960;82:35-45.

[11] Lotfi N, Zomorodi H, Landers R. Thermal Management and Voltage Stabilization in Air-Forced Open-Cathode Fuel Cells. ASME 2015 Dynamic Systems and Control Conference American Society of Mechanical Engineers 2015:V002T26A005.

[12] Wan EA , Van Der Merwe R. The unscented Kalman filter for nonlinear estimation. IEEE Symposium on Adaptive Systems for Signal Processing, Communications, and Control. 2000:153.

[13] Arulampalam MS, Maskell S, Gordon N, Clapp T. A tutorial on particle filters for online nonlinear/non-Gaussian Bayesian tracking. IEEE Transactions on Signal Processing, 2002;50:174188.

[14] Rubenstein C. An Analysis of Tool Life Based on Flank-Face Wear-Part 1: Theory. Journal of Engineering for Industry 1976;98:221-226. 